Fonagy, P; Lemma, A; (2012) Does psychoanalysis have a valuable place in modern mental health services? Yes. BMJ , 344: e1211 10.1136/bmj.e1211

\title{
COMMENT
}

\section{Does psychoanalysis have a valuable place in modern mental health services? Yes}

\section{Peter Fonagy and Alessandra Lemma}

Department of Clinical, Educational and Health Psychology, University College London, London WC1E 6BT, UK; Anna Freud Centre, London; Psychological Therapies Development Unit, Tavistock and Portman NHS Foundation Trust, London; Psychological Interventions Research Centre, University College London

Psychoanalysis is under greater attack than ever before. An unprecedented decommissioning of psychoanalytic services has taken place across the United Kingdom's National Health Service (for example, Forest House NHS Psychotherapy Clinic), justified by cost savings. What are the reasons for this attack and what can be said in psychoanalysis's defence?

It has been claimed, perhaps fairly, that psychoanalysis and psychodynamic psychotherapies have failed to promote a culture of systematic evaluation and that the outcomes are difficult to measure and demonstrate. Relative to the number of studies on the effectiveness of cognitive behavioural therapy, few adequate studies are available of psychodynamic therapy outcomes. A growing body of studies, however, report that psychodynamic therapy is effective in the treatment of both mild and complex mental health problems. For example, a meta-analysis found substantial effect sizes in randomised controlled trials of long term psychodynamic psychotherapy, larger than those for short term therapies.[1] Positive correlations were also seen between outcome and duration or dosage of therapy. Another metaanalysis found that psychotherapy in addition to antidepressants significantly reduced depressive symptoms compared with antidepressants alone.[2] A third meta-analysis found that short term psychodynamic psychotherapy may be more effective than other therapies for somatic disorders.[3] So evidence is on its way.

Convergent support for psychoanalytic approaches comes from 20 studies of brain function changes after a range of psychotherapeutic treatments, including psychodynamic ones, for several mental disorders. [4] Brain changes that have been shown include a substantial increase in $5-\mathrm{HT}_{1 \mathrm{~A}}$ receptor density in patients with major depressive disorder after psychodynamic therapy-this was not the case in patients who received fluoxetine[5]—and normalisation of neuronal activity in patients with somatoform disorders.[6] Ultimately these investigations will enable us to better understand the therapeutic mechanisms of a range of approaches and provide badly needed improvements in our treatments of complex disorders. 
The psychoanalytic approach makes three valuable and unique contributions to a modern healthcare economy. First, in their applied form, psychoanalytic ideas can support mental health staff to provide high quality services despite the interpersonal pressures to which they are inevitably exposed when working with disturbed and disturbing patients. Psychoanalytic understanding helps us to respond in humane ways when anxiety and stress threaten our ability to contemplate behaviour in terms of underlying mental states. The framework psychoanalysis provides for understanding why things go wrong in therapeutic relationships draws on a welldeveloped theory of interactional process.[7] There are few viable alternative models for how a disturbed individual or community can affect the thinking and behaviour of those engaged with them.

Secondly, there are increasingly strong indications that adult mental health problems are developmental in nature; three quarters can be traced back to mental health difficulties in childhood, and $50 \%$ arise before age 14 years.[8] The psychoanalytic model is unique in proposing a developmental theory (of attachment relationships) that is now firmly supported by evidence.[9] It therefore allows us to understand the relationship between early experience, genetic inheritance, and adult psychopathology. This developmental framework emphasises early intervention and has been critical in shaping positive mental health policy, including the UK government's "No Health Without Mental Health" strategy.[10] Acknowledging the developmental, relational foundations of mental health also has important implications for prevention.

Thirdly, psychoanalytical ideas continue to provide the foundations for a wide range of applied interventions. Research and clinical observation show that other modalities - particularly cognitive behavioural therapy_have made use of theoretical and clinical features of the psychoanalytic approach and incorporated these into their techniques. This may well enhance the overall effectiveness of these modalities; for example, some evidence suggests that the good outcomes achieved by other therapies correlate with the extent to which those therapies use psychodynamic techniques.[11]

Research clearly shows that there is no one size fits all approach to the treatment of mental health problems; irrespective of brand, psychotherapy only substantially helps around $50 \%$ of referred patients who complete treatment[12] and medication fares no better.[13] Rationally designed services should therefore provide a range of approaches for which some evidence of effectiveness exists, and should continue to broaden the research base to ensure monitoring and improvement of the effectiveness of these services. More comprehensively, perhaps, than any other theory of the mind, psychoanalysis points to key psychological phenomena and processes (such as the limitations of consciousness, defences, resistance to treatment, transference and countertransference). These have to be integrated into our understanding of clinical work if adequate and effective psychological treatment is to be offered. If psychoanalysis is thrown out, these aspects of the mind will have to be rediscovered-just like Greco-Roman culture was rediscovered after the dark ages.

\section{References}


Leichsenring F, Rabung S. Long-term psychodynamic psychotherapy in complex mental disorders: update of a meta-analysis. Br J Psychiatry 2011;199:15-22.

2.

Jakobsen JC, Hansen JL, Simonsen E, Gluud C. The effect of adding psychodynamic therapy to antidepressants in patients with major depressive disorder. A systematic review of randomized clinical trials with meta-analyses and trial sequential analyses. J Affect Disord2011, published online 16 Apr.

3.

Abbass A, Kisely S, Kroenke K. Short-term psychodynamic psychotherapy for somatic disorders. Systematic review and meta-analysis of clinical trials. Psychother Psychosom 2009;78:265-74.

4.

Karlsson H. How psychotherapy changes the brain. Psychiat Times 2011;28:8.

5.

Karlsson H, Hirvonen J, Kajander J, Markkula J, Rasi-Hakala H, Salminen JK, et al. Research letter: psychotherapy increases brain serotonin 5-HT1A receptors in patients with major depressive disorder. Psychol Med 2010;40:523-8.

6.

De Greck M, Scheidt L, Bölter AF, Frommer J, Ulrich C, Stockum E, et al. Multimodal psychodynamic psychotherapy induces normalization of reward related activity in somatoform disorder. World J Biol Psychiatry 2011 Jun;12:296-308.

7.

Lemma A, Target M, Fonagy P. Dynamic interpersonal therapy: a clinician's guide. Oxford University Press, 2011.

8.

Kim-Cohen J, Caspi A, Moffitt TE, Harrington H-L, Milne BJPR. Prior juvenile diagnoses in adults with mental disorder: developmental follow-back of a prospective longitudinal cohort. Arch Gen Psychiatry 2003;60:709-17.

9. 
Cassidy J, Shaver PR, eds. Handbook of attachment: theory, research, and clinical applications. 2nd ed. Guilford, 2008.

10.

Department of Health. No health without mental health: a cross-government mental health outcomes strategy for people of all ages. 2011.

www.dh.gov.uk/en/Publicationsandstatistics/Publications/PublicationsPolicyAn dGuidance/DH 123766.

11.

Shedler J. The efficacy of psychodynamic psychotherapy. Am Psychologist 2010;65:98-109.

12.

Fonagy $P$. The changing shape of clinical practice: a comprehensive narrative review. Psychoanalytic Psychother2010;24:22-43.

13.

Fournier JC, DeRubeis RJ, Hollon SD, Dimidjian S, Amsterdam JD, Shelton $R C$, et al. Antidepressant drug effects and depression severity: a patient-level meta-analysis. JAMA2010;303:47-53. 